\title{
Zeitwettbewerb deutscher Free-TV-Anbieter
}

\author{
Christian Zabel
}

Der vorliegende Aufsatz untersucht, inwieweit sich die Programmentwicklung im Fernseben mit den zentralen Triebkräften des Wettbewerbs nach Porter erklären lässt. Auf Basis von Interviews mit Branchenexperten sowie Fallstudien aus der deutschen Daytime erläutert die Analyse dabei, wie die Veränderungen in der Nachfrage (Zuschaner, Werbeindustrie) und im Angebot der Programmmärkte die Sender fortlaufend dazu zwingen, ibr Programmschema zu erneuern. Da sich die Senderkonkurrenz somit als TimingWettbewerb interpretieren lässt, wird das Portersche Modell um die zeitliche Dimension erweitert: Lohnt es sich für die Sender, ein Format neu zu entwickeln oder eine bereits erfolgreiche Sendung zu adaptieren? Die weitere Untersuchung deutet dabei auf nur schwache Pioniervorteile hin, da das Feblschlagrisiko hoch und ein Patentschutz kaum ausgeprägt ist. Des Weiteren kann ein Image-Bonus für Pionierformate exemplarisch nicht nachgewiesen werden; vielmehr scheint die quantitative Akzeptanz der Angebote wichtiger zu sein - unabhängig vom Zeitpunkt des Markteintritts. Insgesamt spricht die Analyse für eine „Strategie des schnellen Zweiten“-mit Ausnabme des imageprägenden Bereichs der Event-Sendungen.

Keywords: Programmwettbewerb, Innovation, Imitation, Zeitwettbewerb, TimingStrategien

\section{Einleitung}

„Big Brother“, „Wer wird Millionär?“ oder „Barbara Salesch“ sind einige der Aushängeschilder diverser Format-Wellen ${ }^{1}$, die in den letzten Jahren auf deutschen Fernsehschirmen zu sehen waren. Dabei sind die genannten Sendungen nur ein Symptom für die generelle Beschleunigung des Wettbewerbs unter TV-Sendern. Mit der Schaffung des dualen Systems hat nicht nur das Programmangebot und dessen Akzeptanz quantitativ stark zugenommen, auch die Dynamik hat sich deutlich erhöht: Immer mehr Formate haben eine immer kürzere Lebensdauer. ${ }^{2}$

Dies ignorieren die meisten medienökonomischen Analysen. ${ }^{3}$ Sie begreifen TV-Un-

1 Nach Meckel bezeichnet ein Fernsehformat ein „in seinem Inhalt, seiner Binnenstruktur und seiner Präsentation auf ein klar definiertes Zuschauersegment und einen in die Sendestruktur eingebetteten Programmplatz abgestimmtes mehrteiliges Sendekonzept.“ (Meckel 1997: 478) Darunter fallen etwa Quizshows wie „Wer wird Millionär?“ oder die Gerichtssendung „Barbara Salesch“, ebenso fiktionale Serien. (Zur Problematisierung des Begriffs im Dokumentarischen vgl. Wolf 2003). Der Begriff „Sendung“ wird im Nachfolgenden synonym gebraucht. Mit „Programm“ wird hingegen in diesem Artikel das gesamte Inhalteangebot eines TV-Senders und nicht eine einzelne Sendung bezeichnet.

2 So zählte das Hamburger MMM-Formateregister allein im Jahre 2000423 neue Formate (o. V. 2001a).

3 Vgl. etwa Litman/Shrikhande/Ahn (2000), für den deutschen Markt Holtmann 1998, 1999, Becker/Geisler 1998 und Winter 1999, die den Produktwettbewerb zwar streifen, aber eigentlich von standardisierten Produkten ausgehen. 
ternehmen meist als Verwertungskette, in der festgelegte Produkte ${ }^{4}$ möglichst effektiv (d. h. durch standardisierte Prozesse in Programmplanung, Rechtebeschaffung, Marketing etc.) distribuiert werden. Die statische Effizienz - die Betrachtung von Preisen, Skaleneffekten, Markteintrittsbarrieren etc. - rückt damit in das Zentrum. Der Hauptaktionsparameter, also die Konkurrenz durch Inhalte, bleibt hingegen ein blinder Fleck. Dabei wird die Ausklammerung des Produktwettbewerbs mit einer Standardformel der Medienkritik begründet: Es gebe ohnehin nur „More of the same“, eine Betrachtung der Inhalte erübrige sich daher. ${ }^{5}$ Dies überrascht umso mehr, als, wie William Baumol kürzlich nochmals unterstrich, der Wettbewerb in den Oligopolen moderner Marktwirtschaften in erster Linie über neue Produkte und Prozesse geführt wird: „Innovation has replaced price as the name of the game" (Baumol 2002: 4).

Der vorliegende Artikel untersucht daher, welche Parameter die Formulierung der Produktstrategien von TV-Sendern im Programmwettbewerb beeinflussen. ${ }^{6}$ Die Betrachtung konzentriert sich dabei auf die Konkurrenz im Bereich privater Free-TV-Sender. ${ }^{7}$

Als Rahmenkonzept wird auf die Wettbewerbstheorie von Porter zurückgegriffen, nach der fünf Triebkräfte die Intensität der Konkurrenz (gemessen an der Rendite) in einer Branche bestimmen: die Verhandlungsmacht der Abnehmer, die Verhandlungsstärke der Lieferanten und die Rivalität unter den bestehenden Unternehmen; hinzu kommen die Bedrohung durch potenzielle neue Konkurrenten sowie neue Ersatzprodukte (Porter 1999: 34). Die letzten beiden Aspekte sollen hier vernachlässigt werden: So erscheint ein De-Novo-Markteintritt in dem gut besetzten deutschen Fernsehmarkt für Vollprogramme als wenig wahrscheinlich (Berg/Rott 2000). ${ }^{8}$ Die langfristig kontinuierliche Zunahme der Sehdauer zeigt zudem, dass die Bedrohung durch Ersatzprodukte trotz aller Konvergenz-Erwartungen mittelfristig begrenzt bleibt (Arbeitsgemeinschaften der ARD-Werbegesellschaften 2002: 70).

Zunächst soll geklärt werden, wie die drei zuerst genannten Triebkräfte die Strategieformulierung beeinflussen. Inwieweit veranlassen Änderungen in der Nachfrage und im Programmangebot die Sender zur Entwicklung neuer Produkte? Welche Rolle spielt

4 Mit „Produkt“ sind hier Sendungen gemeint. Zwar ist das gesamte Programm durch die Sendermarke verbunden (z. B. RTL oder SAT.1), allerdings konsumiert der Zuschauer einzelne Sendungen und keine gesamten Kanäle. Er ist nicht gezwungen, sich für oder gegen das gesamte Programm zu entscheiden. Daher ist für eine wettbewerbsstrategische, zuschauerbezogene Betrachtung zunächst die Konkurrenz der einzelnen Formate von Bedeutung.

5 Als beispielhaft kann die von Rust diagnostizierte „nuancierte Imitation“ gelten (Rust 1988: 611), vgl. auch Neumann 1991, Gitlin 1994.

6 Nicht betrachtet wird hier die Produktionswirtschaft, die als Lieferant der Sender anderen strategischen Problemen gegenüber steht.

7 Es wird unterstellt, dass sich die Sender in erster Linie an ökonomischen Erfolgskriterien orientieren. Daher bleibt das öffentlich-rechtliche Fernsehen als Akteur außen vor, da es durch seinen Funktionsauftrag eine deutlich andere Aufgabenstellung hat. Allerdings werden die öffentlich-rechtlichen Sender bei der Betrachtung des Wettbewerbsumfeldes berücksichtigt: Auch wenn sie andere Ziele verfolgen, können sie in einigen Genres eine Konkurrenz zu den privaten Sendern darstellen.

8 Dies gilt auch im Hinblick auf den Kauf der ProSiebenSAT.1-Gruppe durch den amerikanischen Investor Haim Saban. So ist in der Folge über eine strategische Neuorientierung der bestehenden Sender des Unternehmens spekuliert worden, allerdings sind durch den Inhaberwechsel keine neuen Sender in den Markt eingetreten. 
die Produktplanung der Konkurrenten? Ist die grundlegende Bedeutung neuer Sendungen erkannt, stellt sich zudem die Frage des Zeitwettbewerbs: Lohnt es sich, mit einem Pionierformat zu starten oder lieber als Zweiter an den Markt zu gehen?

Methodisch basieren die Ergebnisse auf Fallstudien zu nichtfiktionalen DaytimeSendungen sowie auf Ergebnissen aus 17 Leitfadeninterviews mit hochrangigen Entscheidern aus der TV-Branche (Liste der Interviewpartner siehe Anhang). Die durchschnittlich 60 Minuten dauernden Gespräche wurden zwischen Januar und März 2003 persönlich vom Verfasser geführt; drei Interviews fanden telefonisch statt. Der Leitfaden umfasste sechs Punkte, die jeweils mit mehreren Teilfragen erforscht wurden. Neben zwei explorativ angelegten Teilen zur Abgrenzung von Innovation und Imitation sowie zum Prozess der Formatentwicklung wurden in Anlehnung an die Wettbewerbstheorie von Porter die Branchenkonkurrenz, die Ebene der Lieferanten und die Verhandlungsmacht der Abnehmer untersucht. Der letzte Punkt ging dann noch einmal dezidiert auf die zeitliche Komponente des Senderwettbewerbs ein.

\section{Nachfrage als Determinante der Programmplanung}

Die Zuschauernachfrage im TV-Markt ist sehr flexibel: Die Zuschauer verfügen mittels Fernbedienung und Programmzeitschriften über einen guten Marktüberblick; sie können zudem bei geringen Transaktionskosten zwischen den Programmen wechseln. ${ }^{9}$ Auch wenn umstritten ist, inwieweit die von der GfK gemessenen Daten die tatsächlichen Präferenzen der Bevölkerung widerspiegeln, bilden diese doch die Datengrundlage für die zweite Abnehmergruppe, die Werbewirtschaft. Wie die Entwicklung der Tausender-Kontakt-Preise zeigt, kann diese ihre Präferenzen gut durchsetzen, zumal ihre Nachfrage durch Mediaagenturen stark gebündelt wird (Rott 2003: 249). Angesichts dieser Verhandlungsmacht versuchen die Sender, Nachfrageveränderungen in ihrer Programmstrategie zu antizipieren. Als zentrales Planungsinstrument hat sich dabei das Lebenszyklusmodell etabliert. Der Ansatz geht davon aus, dass ein neues Format von seinem Start bis zu seinem Verschwinden aus dem Programm typischerweise mehrere Phasen durchläuft. In der ersten Stufe der Markteinführung ist die Nachfrage nach dem Produkt noch relativ schwach, steigt dann aber während der folgenden Wachstumsphase erheblich an. Das Zuschauerinteresse stabilisiert sich dann in der Reife- oder Sättigungszeit. Schließlich sinkt die Nachfrage während der Degenerationsphase stetig ab, es sei denn das Produkt wird erfolgreich relauncht (Kauschke/Klugius 2000: 148). Ist der prognostische Wert des Konzepts auch umstritten (Pethig/Blind 1995: 63), so kann die Validität jedoch ex-post überprüft werden. Am Beispiel der Daily Talkshows kann gezeigt werden, dass die Nachfrage nach Formattypen einem Lebenszyklus unterworfen ist. Zwischen 1992 und 2003 wurden in Deutschland insgesamt 24 solcher Formate ausgestrahlt. Als Daily-Talk gelten dabei alle Sendungen von mindestens $30 \mathrm{Mi}$ nuten Länge, die mindestens vier mal wochentäglich ausgestrahlt werden. Weitere Charakteristika dieser Produktionsform sind, dass sie nicht in Staffelform hergestellt wird, überwiegend zwischenmenschliche Thematiken mit mehreren „alltagsnahen Akteuren" als Protagonisten aufgreift und das Studiopublikum einbindet. Abbildung 1

9 Zwar ist die Fernsehnutzung stark ritualisiert; dies bezieht sich aber in erster Linie auf die Nutzung des gesamten Programmangebots. Auf der Ebene einzelner Formate helfen Planungstechniken wie Audience Flow und Stripping (s. u.) lediglich, die Zuschauerfluktuation zu begrenzen - diese bleibt aber immer noch recht hoch (Zubayr 1999). 
zeigt die Entwicklung der Nachfrage im Talkshow-Genre. Als Datengrundlage galten die von der GfK ermittelten Zuschauerzahlen der Formate während einer natürlichen Programmwoche pro Quartal während des Untersuchungszeitraums. ${ }^{10}$

Abbildung 1: Lebenszyklus Daily Talkshows 1992-2003

Zuschauer in Mio./

Anzahl der Formate

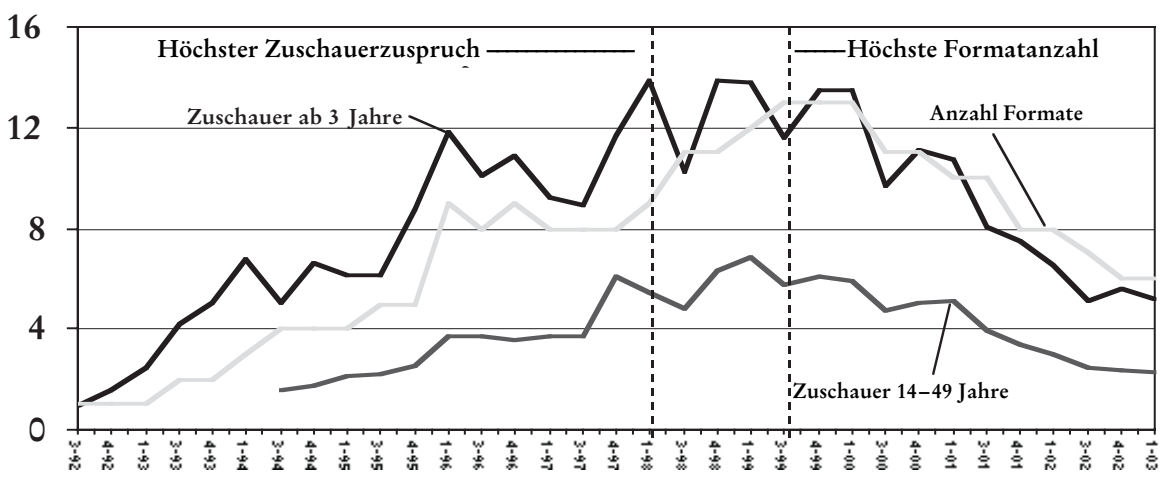

Quelle: Eigene Berechnungen auf Basis von GfK PC\#TV, kumul. Durchschnittsquote einer Programmwoche (pro Quartal, ohne Wiederholungen im Sommer).

Nachdem mit „Hans Meiser“ im September 1992 das erste Format auf Sendung ging, erreichte die Seherakzeptanz dieses Genres im ersten Quartal 1998 ihren Höhepunkt (Abbildung 1). Täglich sahen kumuliert über 14 Mio. Zuschauer Daily Talk-Formate. Jede einzelne Sendung erreichte im Durchschnitt 1,5 Mio. Seher. Im Folgenden nahm die Zahl an Daily Talkshows noch fast zwei Jahre lang zu, obwohl die einzelnen Sendungen bereits immer weniger Seher binden konnten. Die 13 Ende 1999 täglich ausgestrahlten Talkshows erreichten durchschnittlich nur noch 0,9 Mio. Seher. Dabei verloren die Formate, die zunehmend auf früheren, zuschauerschwächeren Timeslots gesendet wurden, auch relativ gesehen an Zuspruch; der durchschnittliche Marktanteil sank von $19 \%$ auf $14 \%$.

Die Veränderungen in dem komplexen Aggregat „Zuschauerpräferenzen“ lassen sich dabei nicht monokausal begründen. Allerdings können mehrere, sich überlagernde Einflussfaktoren ausgemacht werden: Der Grundlegendste und Langfristigste dürfte der gesellschaftliche Wandel sein. Hickethier hat vorgeschlagen, die Veränderungen in den Inhalten des Fernsehens als Modernisierungsprozess im Weberschen Sinne aufzufassen. „Fernsehen ist ein Produkt der gesellschaftlichen Modernisierungen und zugleich Transmissionsriemen“ (Hickethier 2000: 19). So hat sich zum Beispiel die Rolle der Frauen und die Häufigkeit der Frauenrollen im Zeitablauf geändert, bis hin zu der Entwicklung, den „Beruf“ des Fernsehkommissars (fast) ganz in weibliche Hände zu geben

10 Dazu wurde auf die von Krüger vorgeschlagenen „natürlichen Programmwochen“ zurückgegriffen; wo dies nicht möglich war (z. B. ab 2001), wurden eigene Programmwochen ausgewählt (Krüger 2000, 2001). 
(Hesse 2002). Außerdem hat die Reflexivität der Fernsehsendungen stark zugenommen, was als Zeichen der Reife gewertet werden kann (Schmidt 2000). Der gesellschaftliche Wandel kann bestimmte Formate sogar „unmöglich“ machen, so Axel Beyer, Unterhaltungschef des WDR: „Die 90-minütige Samstagabendshow, der berühmte Familienabend, ist deswegen tot, weil es die klassische Familie nicht mehr gibt" (o.V. 2002: 54).

Neben diesen grundlegenden Entwicklungen können noch kurzfristigere, volatile Tendenzen in der öffentlichen Meinung, der "gesellschaftlichen Großwetterlage“ (UFA-Entertainment Geschäftsführer Harry Goering) identifiziert werden, die in der Wahrnehmung der Fernsehmacher eher zyklischer Natur sind. Die meisten Befragten waren sich darüber einig, dass in „schweren“ Zeiten „leichte“ Stoffe größere Erfolgschancen hätten. Dies deckt sich mit der von Ludwig vorgeschlagenen Dichotomie der Medien von Wirklichkeitssuche und -flucht (Ludwig 1998: 156).

Neben diesen exogenen Veränderungen können sich auch endogen Nachfrageeffekte kumulieren und nachfolgend auch konterkarieren. Nach der Konsumkapitaltheorie von Stigler/Becker (Stigler/Becker 1977) nimmt der Nutzen aus dem Konsum bestimmter Güter mit der Zahl der Nutzer zu (Bandwaggon-Effekt), sinkt jedoch wieder, wenn der aggregierte Konsum eine bestimmte Schwelle überschreitet (IndividualitätsEffekt). Das Zusammenspiel dieser Effekte erlaubt auch eine Erklärung scheinbar sprunghafter Nachfrageverläufe in Form eines „deterministischen Chaos“, wie Hofmann und Rauscher beispielhaft für den Modemarkt aufzeigen (Hofmann/Rauscher 1991). Als dritter Faktor kann hier ein Sättigungseffekt angeführt werden - nach der Befriedigung eines Bedürfnisses werden andere vorrangig (Pethig/Blind 1995: 67).

Dieser Sättigungseffekt wird durch selbstreferentielle Effekte auf der Angebotsseite verstärkt. So ist für den einzelnen Manager das Risiko einer Entscheidung geringer, wenn er sich damit im allgemeinen Branchentrend befindet (Blum 1995: 32); wie Borris Brandt, Geschäftsführer Endemol Deutschland, betont, ist dies gerade im Hinblick auf die kurze Laufzeit der Managerverträge von Bedeutung. Des Weiteren kann vermutet werden, dass - in Analogie zu den von Bourdieu betonten Feldeffekten journalistischer Produktion - auch die in der Branche beschäftigten Manager versuchen, von Ihresgleichen anerkannt zu werden (Bourdieu 1998: 105). Hier kann es sich aus karrierestrategischen Gründen als sinnvoll erweisen, dem allgemeinen Branchentrend zu folgen. Schließlich sind Vorschläge, die sich auf Referenzen beziehen können, nach Ansicht der Interviewpartner intern auch leichter durchzusetzen; dieser Zwang wird durch den Werbemarkt verstärkt, der mit seinen Einbuchungen ebenfalls den Formattrends folgt (zu einer ähnlichen Schlussfolgerung für den amerikanischen Markt kommt Kennedy 2002).

So kumuliert sich die Nachfrage; man „versucht, von Erfolgen Dubletten zu machen“ (Christiane Ruff, Geschäftsführerin von Sony Pictures Entertainment). Eine Abstimmung unter den Sendern findet dabei nicht statt. Diese typische Gefangenen-DilemmaSituation führt zu einem Überangebot, das nach Ansicht von Norbert Schneider, Direktor der Landesanstalt für Medien Nordrhein-Westfalen (LfM), in einer „Selbstkannibalisierung im Boom“ münde. Das Über-Angebot verkürze darüber hinaus auch die Formatzyklen - gerade im nicht-fiktionalen Bereich, da etwa Spielschemata schneller erlernt werden. Ein Beispiel aus der jüngeren Vergangenheit ist etwa der Boom der Reality-Shows - und ihr ebenso schnelles Verschwinden. Die scheinbar von diesen Lebenszyklen nicht betroffenen Formate - wie zum Beispiel die Show „Wetten, dass...?“ - sahen die Interviewpartner durchweg als Ausnahme an. Ihr dauerhafter Erfolg wird auf die Beliebtheit einzelner Moderatoren zurückgeführt oder dass so das Lebensgefühl einer bestimmten Alterskohorte getroffen wird, die mit dem Format auch altert. 


\section{Einfluss des Programmangebots auf die Senderstrategie}

Neben der Orientierung an der Nachfrage können neue Formatangebote als zweiter wesentlicher Einflussfaktor bei der Programmplanung angesehen werden. Die Initiative hierzu geht sowohl von den Sendern direkt (von einzelnen Redakteuren, Entwicklungsredaktionen oder aus der Programmbeobachtung) als auch von den Produktionsfirmen aus. Beide Seiten verfolgen in der Produktentwicklung einen marktorientierten Ansatz: Neue Formate werden in der Regel für die problematischen Timeslots der Sender entwickelt. Mit dem Sendetermin kann dann das gegebenenfalls maximal zur Verfügung stehende Budget errechnet werden, z. B. über eine Potenzialanalyse (vgl. Höltich 1994). Eine Einschränkung der potenziell interessanten Genres ergibt sich aus der Markenidentität des TV-Senders und seiner Positionierung im Zuschauermarkt. Zudem kann sich hier die Marktstruktur der Lieferantenebene auswirken. So stellt Röper eine zunehmende Konzentration in der Produktionsbranche fest und äußert die Vermutung, dass Formatangebote von Produzenten, die zu dem gleichen Konzern wie der Sender gehören, bevorzugt werden (Röper 2000, Pätzold/Röper 2002). Während die befragten freien Produzenten die Vermutung von Röper aus ihrer eigenen Praxis unterstrichen, dementierten dies die Vertreter von Sendern und konzerneigenen Produktionsfirmen.

Als Ideenquellen für neue Formate nannten die Interviewpartner die

- internationale Marktbeobachtung,

- Adaption von Buch- oder Kinotiteln,

- maßgeschneiderte Entwicklung für bestimmte Comedians/Moderatoren,

- Spin-Offs etablierter Marken und schließlich die

- persönliche Umwelt der Entwicklungsredakteure.

Die Attraktivität dieser Formatideen für den einzelnen Sender kann dann grob anhand der Dimensionen Risiko, Leistung und Kosten eingeschätzt werden (Winter 1999: $221 \mathrm{ff})$.

\subsection{Risiko als zentrale Größe}

Die Einschätzung der Verwertungschancen eines neuen Formats ist wesentliches Entscheidungskriterium der Programmplanung: Zwei Drittel der neu gestarteten Sendungen werden bereits nach einem Jahr Laufzeit abgesetzt, da sie nicht die jeweiligen Quotenerwartungen des Managements erfüllen (Bughin 2000: 14). Auf der Ebene einzelner Produkte wird daher versucht, diese Unsicherheit durch Qualitätstests im Vorfeld zu senken. Daraus resultiert ein „Formatting“-Effekt - also der Trend, Sendungen „nach bestimmten kommerziell erfolgversprechenden Mustern zu gestalten“, was radikale Entwürfe seltener mache (Meckel 1997: 479).

Neben dieser Reaktion auf das produktimmanente Risiko versucht die Programmplanung zudem, über die Steuerung des gesamten Sendungsportfolios der Unsicherheit in der Unternehmensumwelt zu begegnen. Wie Litman, Shrikhande und Ahn für den US-amerikanischen Markt zeigen, erzielen neue Formate in der Regel nur unterdurchschnittliche Einschaltquoten. Gleichzeitig verringert eine solche (thematische) Diversifikation aber die Anfälligkeit des gesamten Programmangebots für unvorhergesehene Änderungen, z. B. auf den Angebotsmärkten (Litman/Shrikhande/ Ahn 2000). Ähnlich kommen Miller und Shamsie in ihrer Analyse der amerikanischen Kino-Industrie zu dem Schluss, dass die Hollywood-Majors bei zunehmender Um- 
weltunsicherheit ihre Produktpaletten eher diversifiziert haben, um für die sie umgebende Turbulenz besser gerüstet zu sein (Miller/Shamsie 1999: 113f.).

Die Risikobereitschaft variiert dabei zudem mit der Wettbewerbsposition der Sender. Das Risk-Return-Paradoxon beschreibt die Beobachtung, dass Unternehmen in Krisensituationen oft $\mathrm{zu}$ riskanteren Innovationsprojekten tendieren; bei gut laufenden Geschäften hingegen bleibt die Unternehmenspolitik eher konservativ (Brockhoff 1999: 107). Dies bestätigten die meisten der befragten Interviewpartner. So vermutet Typhoon Networks-Geschäftsführer Marc Conrad, dass RTL seine Marken auf hohem Niveau weiterentwickeln kann, während Konkurrent SAT.1 dort riskantere Projekte angehen müsse. Schließlich ist noch anzufügen, dass die Einschätzung der Ungewissheit - ganz im Schumpeterschen Sinne - auch auf die persönliche Risikobereitschaft der Entscheider zurückzuführen ist.

\subsection{Leistung neuer Sendungen}

Die Leistung, also die (wahrgenommenen) Produkteigenschaften sind der zweite Faktor bei der Beurteilung der Attraktivität (und damit auch des Risikos) neuer Formate. Hier muss allerdings einschränkend festgehalten werden, dass sich die Leistung eines Formats für die Rezipienten nicht objektiv feststellen lässt, was im Wesentlichen an der beschriebenen Komplexität und Dynamik der Fernsehrezeption liegt. So können allenfalls allgemeine, produktunspezifische Erlebnisfaktoren von Sendungen identifiziert werden (Dehm/Storll 2003).

Sind die Leistungskriterien also nicht letztgültig operationalisierbar, so kann aufgrund der Expertenbefragung doch vermutet werden, dass zumindest branchenintern ein weit reichender Konsens über kritische Erfolgsfaktoren neuer Formate existiert. Deren Gewichtung kann allerdings je nach konkretem Format erheblich variieren. Als ausschlaggebende Aspekte identifizierten die befragten Experten

- die Stringenz und Kohärenz des Sendungskonzepts,

- die Qualität der beteiligten Schauspieler/Moderatoren („Stars“),

- die weiteren kreativen Beteiligten (vor allem der Producer und Autoren),

- die Existenz ähnlicher Formate im Ausland,

- der Production Value als Sichtbarmachung des Budgets,

- das Marketing sowie

- die investierte Entwicklungszeit (und damit -geld).

Neben dem Erfolg im deutschen Markt hat in letzter Zeit auch die internationale Vermarktbarkeit als Leistungskriterium an Bedeutung gewonnen. So erzielen deutsche Unternehmen mittlerweile umfangreiche Einkünfte durch den Verkauf von Serien, Filmen und Dokumentationen ins Ausland. ${ }^{11}$ Allerdings stellt die Attraktivität für den deutschen Markt immer noch die zentrale Erfolgsgröße dar, zumal deutsche Produktionen nach Aussagen der Befragten nicht in dem gleichen Maße vermarktbar sind wie etwa USamerikanische Formate. ${ }^{12}$ Schließlich ist - nicht zuletzt nach dem Erfolg der Castingshows - die Einbeziehung zusätzlicher Erlösquellen (durch Telefonanrufe, Plattenver-

11 Die Einkünfte aus dem europäischen Formathandel werden auf 500 Mio. € geschätzt (Mann 2004).

12 Dies liegt unter anderem an der Sprache, dem Cultural Discount und dem in der Regel geringeren Produktionsbudget im deutschen Raum. Kooperationen sind hier nur für einige Genres ein Ausweg, z. B. für Dokumentationen. 
käufe, Merchandising, aber auch Product Placement etc.) wichtiger geworden. Können hiermit auch substantielle Erlöse erzielt werden, bleibt die Attraktivität des Formats immer noch die zentrale Leistungsgröße, da ohne eine entsprechende Reichweite keine nennenswerten Nebenerlöse erwirtschaftet werden können.

\subsection{Relevanz der Kosten neuer Formate}

Schließlich beeinflussen die Kosten eines Formats dessen Attraktivität. Aufgrund der geringen Fertigungstiefe der Sender besitzen die Materialkosten der Sendungen eine starke Hebelwirkung: Geringere Aufwendungen für Inputfaktoren schlagen direkt auf den Gewinn durch (etwa im Gegensatz zu Branchen, wo immer fixe Kosten für die Weiterverarbeitung anfallen). Dies legt nahe, dass die Sender eher versuchen, die Produktionskosten zu drücken, um den Gewinn zu steigern, anstatt zu versuchen, mit aufwändigen Produkten den Umsatz zu erhöhen (Winter 1999: 106). ${ }^{13}$ Neben dem vereinzelten Rückgriff auf „Rabattmarken“ (Sabine Eckhard, Head of Serial Production, UFAFernsehproduktion $\mathrm{GmbH}$ ), wie etwa der Verpflichtung günstigerer Schauspieler, lassen sich substantielle Kosteneinsparungen allerdings nur durch den Rückgriff auf weniger aufwändige Produktionstypen erreichen. So konzentrierte sich zum Beispiel SAT.1 jüngst auf tägliche Krimiserien mit Doku-Anmutung in der Access-Prime-Time, die erheblich schneller und günstiger zu produzieren sind (Butzek 2003). Allerdings sind auch die Formatkosten verschiedener Genres mittel- und langfristig deutlichen Schwankungen unterworfen, die aus der Senderkonkurrenz resultieren. Kruse vermutet etwa, dass die öffentlich-rechtlichen Sender durch ihre Nachfrage die Preise für positionale Faktoren (Moderatoren, Sport-Übertragungsrechte) in die Höhe treiben (Kruse 2000: 14). Der gegenteilige Fall des nachlassenden Interesses wurde jüngst am Preisverfall der Bundesligarechte deutlich.

\section{Branchenkonkurrenz als weitere Einflussgröße}

Neben Angebot und Nachfrage ist der Wettbewerb unter den TV-Sendern die dritte Triebkraft in der Branche. Die Rivalität kann aus drei Gründen als hoch eingeschätzt werden: Erstens ist, wie gezeigt, die Zuschauer- und Werbemarktnachfrage sehr flexibel. Zum Zweiten liegt in der Rundfunkproduktion eine starke Fixkostendegression vor (Heinrich 1999: 120). Von daher ist es für den einzelnen TV-Sender grundlegend rational, die eigene Einschaltquote zu maximieren und sich nicht mit den Konkurrenten abzustimmen. Drittens überschneiden sich die anvisierten Zielgruppen trotz unterschiedlicher Marktpositionierungen der Sender stark - zumindest bei den Vollprogrammen. Die Spezialisierung der auf den breiten Zuschauermarkt zielenden Programme bleibt sehr begrenzt, wie zahlreiche Beispiele aus der jüngeren Programmgeschichte beweisen; dies macht die Programme anfällig für Wettbewerbsvorstöße der Konkurrenz. ${ }^{14}$

Die Reaktionsverbundenheit unter den Sendern variiert dabei mit der Breite ihrer

13 Diese Überlegung gilt hingegen nicht für Event-Produktionen, die vor allem einen Beitrag zum Image des Senders leisten sollen - und sich eher „indirekt“ über die gestärkte Kanalmarke refinanzieren (siehe unten).

14 Nach dem Erfolg von „Wer wird Millionär?“ haben zum Beispiel alle größeren Sender versucht, mit Imitationen nachzuziehen, ähnliche Entwicklungen konnten nach dem Erfolg von „Big Brother" und den Courtshows beobachtet werden, jüngst wieder bei den Casting-Formaten. 
strategischen Optionen. Diese werden zunächst durch die Kostenposition der Sender begrenzt. Die Herstellung eines TV-Movies kostet zum Beispiel zwischen 900.000 und 1,8 Mio. $€$ (HMR International 2002). Für einen Sender, der im Schnitt nur 4-5\% der Zuschauer erreicht, wird eine solche Produktion über Werbung nicht refinanzierbar sein - auch nicht bei mehrfacher Ausstrahlung und Auslandsverwertung. In dieser Formatkategorie stehen daher zum Beispiel nur die großen Vollprogramme untereinander in der Konkurrenz.

Neben den Kosten grenzt auch die Heterogenität der Zielgruppe die Palette möglicher Formate ein. Kleinere Sender erreichen homogenere Zielgruppen, was „spitzere“, genauer auf das spezielle Publikum zugeschnittene Formate ermöglicht. Solche Neuerungen sind auf breitenorientierte Kanäle kaum zu übertragen - höchstens auf peripheren Programmplätzen (z. B. ist zu bezweifeln, dass das umstrittene MTV-Format "Jackass“ auch außerhalb der begrenzten Seherschaft des Musikkanals erfolgreich wäre, wenn es etwa in der Primetime von ProSieben laufen würde).

Abbildung 2 beschreibt schematisch die Marktinterdependenz ausgewählter Sender, die vor allem innerhalb mehrerer strategischer Gruppen stattfindet. Die „Vollprogramme“ zum Beispiel stehen vor allem untereinander in Konkurrenz. Sie haben sehr heterogene Zuschauerschaften und bieten (fast) die gesamte Breite an möglichen Formaten an. Ein neues Format, das eine solche disperse Zuschauerschaft anspricht (zum Beispiel „Deutschland sucht den Superstar“), setzt die Mitbewerber der Gruppe stärker unter Druck als die anderen Sender, die solche Shows vielleicht überhaupt nicht anbieten können (wie etwa Kabel1 oder arte) bzw. ein anderes Publikum ansprechen (etwa N24). Dies bedeutet nicht, dass die Sender verschiedener Gruppen untereinander überhaupt nicht in Konkurrenz stehen, allerdings sind Wechselwirkungen zwischen Vollprogrammen und Sendern wie RTL2 oder MTV eher punktuell und auf einzelne Genres begrenzt (dies wird durch die räumliche Distanz in der Grafik verdeutlicht).

Natürlich wird die Konkurrenz unter den Sendern wesentlich durch die Konzernzugehörigkeit der Kanäle beeinflusst. So betonte Jobst Benthues, Leiter Unterhaltung ProSieben, dass in der ProSiebenSAT.1-Gruppe die Sendungen aufeinander abgestimmt werden, um Kannibalisierungseffekte zu vermeiden und attraktive Inhalte optimal auf die Sender zu verteilen. ${ }^{15}$ Allerdings erscheint die ausschließliche Reduktion des Wettbewerbs auf Konkurrenz zwischen Sendergruppen als übertrieben. Auch innerhalb der Gruppen besteht - auch wegen der beschriebenen, nicht absolut trennscharfen Positionierung - zum Teil heftige Konkurrenz (z. B. bei Docu-Soaps, Servicesendungen).

15 Dies gilt gerade für Kaufproduktionen wie etwa US-Serien oder Kinofilme. Eine weitere Möglichkeit ist die Verwertung eines Formats auf mehreren Kanälen, wie es die RTL-Gruppe bei der Casting-Show „Deutschland sucht den Superstar“ vorexerziert hat, wo das eigentliche Format auf dem „Hauptsender“ RTL, die begleitenden Formate auf dem kleineren Kanal Vox zu sehen waren (vgl. auch das Splitting bei der zweiten Staffel von „Big Brother“ zwischen RTL und RTL2). 


\section{Abbildung 2: Strategische Gruppen im Formatwettbewerb}

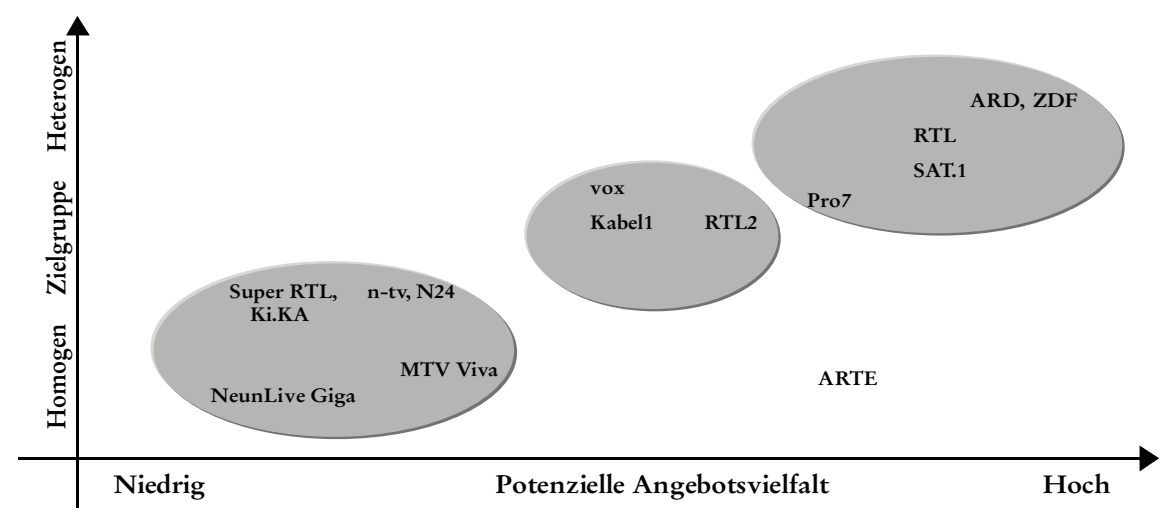

Quelle: eigene, schematische Darstellung, Gruppierung ausgewählter Sender; Angebotsbreite bezieht sich auf Zahl der Genres/Produktionsformen, die der jeweilige Sender anbieten kann (in Anlehnung an Krüger 2001, HMR International 2002).

\section{Programmwettbewerb speziell als Timing-Wettbewerb}

Da die Zuschauerakzeptanz von Formaten mit der Zeit in der Regel deutlich nachlässt, neue Konzepte produktionsseitig fortlaufend angeboten werden und die Sender untereinander grundsätzlich in Konkurrenz stehen, stellt sich nicht so sehr die Frage, $o b$ die Sender neue Formate ins Programm nehmen, sondern wann sie dies tun. Das Portersche Wettbewerbsschema ist um die Zeitdimension zu erweitern, der Programmwettbewerb äußert sich damit in erster Linie als Timing-Wettbewerb. „Ziel von Timing-Entscheidungen ist es, Produktvorteile durch den, richtigen' Markteintrittszeitpunkt in KKVs (komparative Konkurrenzvorteile, C.Z.) zu transformieren oder Nachteilspositionen durch einen Marktaustritt aufzugeben“ (Backhaus 1995: 193). Hier lassen sich drei Strategietypen unterscheiden (Pleschak/Sabisch 1996: 89): Bei der Pionierstrategie versucht der Sender, eine zeitlich begrenzte Monopolstellung zu erringen und sich während dieser Periode für den kommenden Wettbewerb zu rüsten. Für nachher in den Markt eintretende Unternehmen, die so genannten second-to-market, lassen sich zwei generische Strategietypen unterscheiden. Die „Frühen Folger" treten kurz nach dem Pionier in den Markt ein und profitieren damit von dessen Erfahrungen. Der Lebenszyklus des Marktes steht noch am Anfang, und trotz der - in der Regel heftigen - Konkurrenz durch den Pionier sind die Marktpositionen noch nicht endgültig verteilt. Die Unternehmen des Typs „Late-to-market“ werden hingegen erst dann aktiv, wenn sich bereits ein Technologiestandard bzw. ein dominantes Design herausgebildet hat und über die Marktentwicklung und das Käuferverhalten genaue Daten vorliegen.

Im Fernsehmarkt ist dabei die Unterscheidung zwischen Sendungsinnovation (Pionier) und Imitation (Second-to-market) nicht ganz einfach. Einerseits ist jede Sendung ein Unikat, da zwei Sendungen nie vollkommen identisch sind. Andererseits hat im mittlerweile ausdifferenzierten Fernsehmarkt jedes Format einen ähnlichen Vorläufer, was nur noch die vielzitierte "nuancierte Imitation“ (Rust 1988: 611) ermöglicht. Die Unmöglichkeit einer „endgültigen“, juristisch durchsetzbaren Distinktion wird auch im deutschen Formatrecht deutlich (Holzporz 2002: 87). 
In Anlehnung an Kotzbauers Modell der perzipierten Innovationshöhe kann der Neuigkeitsgrad aber im Hinblick auf den Zuschauer definiert werden (Kotzbauer 1992: 230). Dies bedeutet zunächst nur, dass das Format auf dem deutschen Markt neu sein muss. ${ }^{16}$ Darüber hinaus müssen die Fernsehsendungen auch in ihrem zeitlichen Kontext bewertet werden, da kulturelle Produkte - zu denen auch Fernsehformate gehören ihren Novitätsgrad immer aus der Relation zu bestehenden Wertkategorien ihrer Zeit beziehen (Groys 1992: 44). Das Konzept eines Formats, das z. B. das letzte Mal vor 30 Jahren ausgestrahlt wurde, kann für heutige Zuschauer durchaus wieder „innovativ“ sein. Entscheidend für die Abgrenzung ist die Ähnlichkeit in wesentlichen Strukturelementen. So ist etwa für eine Personality-Show (wie etwa „Die Harald Schmidt Show“) nach Ansicht der Branchenexperten vor allem der Charakter und die Funktion des Moderators entscheidend, bei einem Quiz hingegen ist die Struktur (Spielmodus etc.) bedeutender.

\subsection{Mögliche Vorteile von Pionierformaten}

\section{Patentschutz bei Innovationen?}

In der neoklassischen Theorie wird das höhere Produktrisiko und die Entwicklungskosten einer Innovation durch eine zeitlich begrenzte Monopolstellung in dem neuen Markt kompensiert, die in der Regel durch Patente sichergestellt wird. Wie Heinrich ausführt, können die Medienprodukte nur unzureichend gegen Imitation geschützt werden, was zu einer „Herumerfinderei“ führe (Heinrich 2002: 170). ${ }^{17}$ Ein De-facto-Patentschutz wäre dann nur möglich, wenn durch das neu entwickelte Format spezifische, kritische Produktionsfaktoren (z. B. Übertragungsrechte) „belegt“ würden, die für Imitationen unumgänglich sind. Allerdings gelingt es in der Realität kaum einem TV-Sender, solche positionalen Inputs exklusiv zu binden - ein Beispiel hierfür sind etwa die Übertragungsrechte an der Formel 1.

Wenn zunächst (fast) alle Inputfaktoren für die Konkurrenten grundsätzlich verfügbar sind, kommt als einziger, minimaler „Patentschutz“ die Zeit in Frage, die Nachzügler brauchen, eine Innovation zu imitieren. Diese Spanne beruht einerseits auf der Inflexibilität des aktuellen Programmschemas, andererseits auf der Produktionszeit

16 Dagegen spielt es keine Rolle, ob es international ohne Vorläufer ist. Zwar werden international erfolgreiche Produktionen nach Aussage aller Interviewpartner im senderinternen Entscheidungsprozess begünstigt; allerdings bleibt das Innovationsrisiko bestehen. So sind im deutschen Markt - wie in anderen Ländern auch - zahlreiche international erfolgreiche Formate gescheitert - von „The Weakest Link“ (auf RTL als „Der Schwächste fliegt“) über „The Sopranos“ (ZDF), „CA\$H“ (ZDF), „Fame Academy“ (RTL2) bis jüngst zu „The Bachelor“ (RTL), um nur einige zu nennen.

17 Rechte lassen sich in der Regel nur einfordern, wenn das neue Format identisch mit dem Ursprünglichen ist (wie bei dem unter 3.2. erwähnten Formathandel). Allerdings reichen bereits kleine Veränderungen aus, um den Formatschutz effektiv auszuhebeln. Dies zeigen nicht zuletzt die zahlreichen, juristisch folgenlosen Kopierversuche, wie sie in den letzten Jahren auf RTL und SAT.1 zu beobachten waren (etwa im Mai 2004: „Der große Deutsch-Test“ (RTL) und „Der große deutsche Prominenten-Buchstabier-Test“ (SAT.1)). Auch auf internationaler Ebene erweist sich die Durchsetzung als sehr schwierig (Mann 2004) - die Firmen verweisen daher verstärkt auf die Vorteile eines lizenzierten Formats, durch das der Käufer auf Erfahrungen, Skripts, Set \& Technik etc. zurückgreifen kann. 
eventueller „Verfolger“-Sendungen. SAT.1-Geschäftsführer Martin Hoffmann veranschlagt den Zeitbedarf, ein fiktionales Format in der Primetime zu programmieren, auf ein Jahr (o.V. 2001). Die Produktionsdauer liegt nach Einschätzungen der Interviewpartner im non-fiktionalen Bereich zwischen sechs Wochen und sechs Monaten.

\section{Wabl der Marktposition}

Während seiner vorübergehenden Monopolstellung kann der Pionier sich im Hinblick auf die Sendezeit optimal positionieren. So konnte RTL zum Beispiel mit der DailySoap „Gute Zeiten, Schlechte Zeiten“ den attraktiven Timeslot um 19.40 Uhr besetzen, der eine wichtige Lead-In-Funktion für die folgenden Sendungen erfüllt (wegen des bedeutenden Umschaltzeitpunkts 20.15 Uhr) und bei dem bereits hohe Zuschauerzahlen erreicht werden. Die später auf den Markt eintretenden Daily Soaps mussten dann auf andere, frühere Timeslots gesetzt werden, um eine direkte Konkurrenz zu vermeiden. Ähnliches gilt für die Talkshows, bei denen RTL zuerst den zuschauerstärksten Timeslot im Daytime-Bereich besetzte (werktags 16.00 Uhr).

Neben der programmplanerischen kann die Ausrichtung aber auch inhaltlich erfolgen. Als Beispiel hierfür könnte etwa die Courtshow „Barbara Salesch“ gelten, die zuerst als Halbstunden-Format auf dem 18.00 Uhr-Sendeplatz von SAT.1 gezeigt wurde und als Schiedsgericht mit realen Fällen konzipiert war. Mit der Passage auf den 15.00 Uhr-Sendeplatz übernahm das Format eine semi-fiktionale Erzählstruktur und behandelte auch Fälle aus dem Strafrecht, was den Durchbruch brachte (Brunst 2003: 3).

\section{Setzen von Produktnormen}

Als weiterer Vorteil des Produktpioniers gilt, dass das Format Normen setzen kann. Wie Abernathy und Utterback zeigen, dominieren im Lebenszyklus einer Technologie zuerst die Produktinnovationen; mit der Durchsetzung eines dominanten Designs verlagert sich die innovative Tätigkeit dann auf die Prozessinnovationen (Abernathy/Utterback 1975). Insbesondere die erste Phase scheint hier im Hinblick auf den Produktionsvorlauf möglicher Nachfolger von Interesse, da das Pionierformat so Zeit hat, „seine Form zu finden“. Dies ist insoweit von Bedeutung, als im Idealfall (werk-) täglich die Möglichkeit besteht, das Format an die Zuschauerwünsche anzupassen; ein Beispiel ist die bereits genannte Sendung „Barbara Salesch“. Im Bereich der Sitcom gelang es 1997 der damaligen ColumbiaTriStar Film- und Fernsehproduktion mit einer Prozessinnovation (serienähnlicher Dreh auf Film statt Aufzeichnung mit Studioatmosphäre), den Produktstandard zu etablieren und dem Genre zum Durchbruch zu verhelfen (Holzer 1999: 103).

\section{Image und Reputation}

Allgemein wird angenommen, dass Innovationen das Image des gesamten Senders stärken, Imitationen in dieser Hinsicht jedoch negative Auswirkungen haben. Die Untersuchung des Teilmarktes für Daily Talkshows zeigt jedoch, dass die Zuschreibung von Kompetenzwerten in einzelnen Sparten stärker von der Akzeptanz des Angebots abhängt als von der Reihenfolge des Markteintritts. So schrieben die Zuschauer dem Formatpionier RTL in den Erhebungen des ARD/ZDF Winter-Trends zwar absolut die höchsten Kompetenzwerte zu. Setzt man diesen Wert jedoch mit den addierten Wochendurchschnitts-Zuschauerzahlen (Seher ab 3 Jahren) in Verbindung, so zeigt sich, 
dass der Formatpionier RTL relativ gesehen die Zuschauernachfrage nicht viel besser in Kompetenzzuschreibungen umwandeln konnte als etwa SAT.1 oder ProSieben. Noch deutlicher wird dies, wenn man bedenkt, dass RTL im Untersuchungszeitraum 1996-2001 nur ein im Vergleich zu seinen Konkurrenten unterdurchschnittliches Verhältnis Talkshowkompetenz/Zuschauer erreicht. Im Falle eines Pionier-Bonus würde man eher davon ausgehen, dass der Sender als anerkannter First-Mover (das RTL-Format „Hans Meiser“ kam zwei Jahre vor dem ersten Nicht-RTL-Verfolger auf den Markt) hier ein besseres Verhältnis erreicht. Abbildung 3 zeigt die Ergebnisse. ${ }^{18}$

\section{Abbildung 3:Spartenkompetenz Daily Talk im Vergleich zur Zuschauerakzeptanz}

Verhältnis Talkshowkompetenz -

erreichte Zuschauer ab 3 J. in Mio.

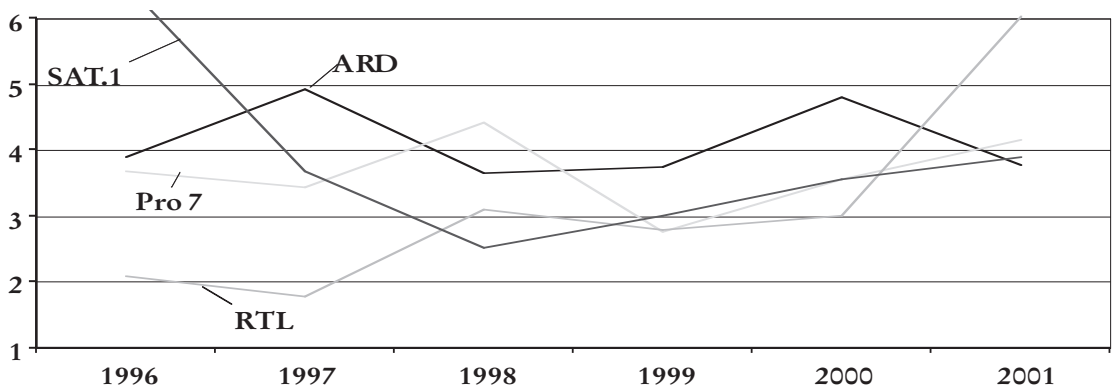

Quelle: Eigene Berechnung auf Basis von GFK PC\#TV, (Zuschauer ab 3 Jahren, Summe der durchschnittlichen Reichweiten von je einer Programmwoche pro Quartal), Kompetenzzuschreibung lt. ARD/ZDF-Wintertrend.

Damit ist nicht gesagt, dass Pioniertormate nicht zur Imagebildung beitragen. Allerdings legt die Untersuchung nahe, dass Regelformate dies eher über einen höheren Markterfolg tun als über die bloße Tatsache, „Erster“ gewesen zu sein. Event-Produktionen, bzw. die Sendungen, die in erster Linie als „Unikate“ konzipiert sind, betrifft dies natürlich nicht. Diese sollen mit erheblichem Herstellungs- und Marketingaufwand die Kompetenz des Senders, Außergewöhnliches zu bieten und Ereignisse zu schaffen, unterstreichen. Dementsprechend hat nach übereinstimmender Einschätzung der Geschäftspartner in jüngerer Vergangenheit die Innovation in diesem Bereich an Bedeutung gewonnen. Der ehemalige SAT.1-Programmgeschäftsführer Martin Hoffmann zum Beispiel sieht Event-Movies wie „Der Tunnel“ als „herausragendes Ereignis“, das eine „positive Grundakzeptanz von SAT.1 im Zuschauerbewusstsein“ verankern helfen soll.

18 Die Ausreißer sind darauf zurückzuführen, dass erstmals Talkshowprogramme angeboten wurden (SAT.1 1996) bzw. die Zahl erheblich verringert wurde (RTL 2001). Im letzten Fall kann von gewissen Trägheitseffekten in der Bewertung durch den Zuschauer ausgegangen werden, die durch die Befragungsmodalitäten verstärkt werden. 


\section{Markentreue}

Wie gezeigt wurde, spricht einiges dafür, dass der Sender aus einer Pioniersituation keine Imagevorteile unabhängig vom Markterfolg des innovativen Formates ziehen kann. Jedoch könnten einzelne Formate während ihrer zeitlich befristeten Monopolstellung Imagevorteile aufbauen und bewahren, so dass sie im späteren Wettbewerb über eine stärkere Kundenbindung verfügen. Pionierformaten wird etwa aus Praktikersicht bescheinigt, sich am ehesten durchzusetzen und das Ende einer Formatwelle zu überleben (Neumann 1991: 141).

Dem während der Monopolstellung aufgebauten Markenwert kommt dabei eine zentrale Bedeutung zu, da emotionale Kompetenzzuschreibungen eine längere Lebenszeit als objektive Produktvorteile haben. Keller hat vorgeschlagen, dies anhand des „Customer-Based Brand Equity“-Modells (CBBE) zu messen. Danach ist der Markenwert aufzufassen als „differential effect that brand knowledge has on consumer response to the marketing of that brand" (Keller 1998: 45). In Niedrigkostensituationen wie der Fernsehprogrammwahl könnte nach Keller bereits die Bekanntheit der Marke gegenüber ihren Mitbewerbern ausreichend für eine Produktwahl sein (Keller 1993: 3). McDowell und Sutherland haben das Konzept auf den audiovisuellen Sektor übertragen und sehen darin eine Erklärung für die unterschiedlich starken Lead-Ins von Formaten (McDowell/Sutherland 2000: 235-238). Pionierformate müssten demnach gegenüber ihren Imitaten einen Imagevorsprung haben, der sich in einem Audience-Flow-Differential niederschlägt. Um eine aussagekräftige Datenbasis zu haben, müssen die untersuchten Formate zur gleichen Zeit an den gleichen Tagen ausgestrahlt werden, im Inhalt nahezu identisch und für die Zuschauer gleichermaßen verfügbar sein sowie nicht durch kurzfristige Promotion-Aktivitäten gefördert werden.

Hier soll exemplarisch der Teilmarkt der Courtshows auf dem werktäglichen 15.00 Uhr-Sendeplatz untersucht werden. Bis September 2002 wurden zwei Formate in dem Timeslot ausgestrahlt: Das öffentlich-rechtliche, mittlerweile eingestellte „Streit um drei“ (Sendebeginn 15.15 Uhr) und die SAT.1-Sendung „Barbara Salesch“. Die beiden Formate sind 1999 fast gleichzeitig als Pioniere auf den Markt gekommen. Im September 2001 zeigte RTL erstmals mit „Das Jugendgericht“ eine Courtshow, allerdings auf dem 16.00 Uhr-Sendeplatz. Am 2. September 2002 ersetzte RTL dann seine auf den 14.00 Uhr und 15.00 Uhr laufenden Talkshows bzw. Füllsendungen durch die Formate „Das Strafgericht“ (14.00 Uhr) und „Das Familiengericht“ (15.00 Uhr). Zu diesem Zeitpunkt war das SAT.1-Format „Barbara Salesch“ der unbestrittene Marktführer. Es erreichte nicht nur weitaus höhere Einschaltquoten als sein öffentlich-rechtliches Pendant, sondern wurde auch professionell hoch geschätzt: Das Format bekam 2002 den „Deutschen Fernsehpreis“. In verschiedenen Umfragen bescheinigten die Zuschauer SAT.1 dank „Barbara Salesch“ eine deutlich höhere Kompetenz in dem Genre als dem ZDF (ZDF 2002: 23, SevenOneMedia 2002: 10).

\section{Markentrene als Widerstandskraft gegen Substitutionseffekte}

Das neue RTL-Format „Das Familiengericht“ hat nur unvollständig eine neue Zuschauerschaft für das Genre „Courtshows“ gewonnen - ein gewisser Teil der Nachfrage wurde lediglich von den Konkurrenzformaten abgezogen (zum Kreations-/Substitutionseffekt: Rott/Schmitt 2001).

So stieg die um die Entwicklung der Sehdauer bereinigte Gesamtzuschauerzahl des Genres (15 Uhr-Sendeplatz) im September - nach Einführung des „Familiengerichtes“ 


\section{Abbildung 4: Kreations- und Substitutionseffekt nach Einfübrung des „Familiengerichts"}

\begin{tabular}{lcc}
\hline Zuschauer Courtshows & Zuschauer ab 3 Jahren & Zuschauer 14-49 Jahre \\
\hline August 2002 & 2,94 & 1,07 \\
September 2002 & 3,83 & 1,54 \\
Differenz August/September & $+0,89$ & $+0,47$ \\
Reichweite „Das Familiengericht“ & 1,56 & 0,81 \\
Von anderen Formaten abgezogene Nachfrage & 0,67 & 0,34 \\
\hline
\end{tabular}

Quelle: Eigene Darstellung auf Basis von GfK PC\#TV, korrigiert um die Entwicklung der Sehdauer.

- im Vergleich zum Vormonat zwar um 0,89 Mio. (Zuschauer ab 3 Jahren) bzw. um 0,47 Mio. (Zuschauer 14-49 Jahre) an. Allerdings hatte das neue Format „Das Familiengericht" eine Reichweite von 1,56 bzw. 0,81 Mio. Sehern. Die bestehenden Formate verloren also mindestens 0,67 Mio. Zuschauer bzw. 0,34 Mio. Zuschauer.

Auf Basis des CBBE-Konzeptes ist zu erwarten, dass das Format mit höherer Wertschätzung von dem Substitutionseffekt weniger stark betroffen ist als die Sendung mit einer niedrigen CBBE. ${ }^{19}$ Wenn die CBBE hingegen keine Rolle spielt, so müssten die beiden Sendungen Zuschaueranteile proportional zu ihrer erzielten Reichweite verlieren. Die Sendung „Streit um drei“ hätte demnach auf Basis der Augustwerte 31 \% der Verluste bei den Zuschauern ab 3 Jahren zu tragen (0,96 Mio. Reichweite dividiert durch 2,94 Mio. Gesamtnachfrage). Da die komplette substituierte Nachfrage sich wie gezeigt auf 0,67 Mio. beläuft, würde dies ein Minus von 0,21 Mio. Zuschauer bedeuten. Für die 14- bis 49-Jährigen ergibt sich ein Verlust von 0,07 Mio. Analog dazu müssten die Reichweiten bei dem Format „Barbara Salesch“ um 0,46 bzw. 0,27 Mio. sinken.

Abbildung 5 zeigt, dass ein CBBE-Effekt nicht nachgewiesen werden kann: Die beiden Formate verlieren Zuschauer exakt proportional zu ihrer vorherigen Reichweite. Auch im Vergleich zu ihren jeweiligen Vorprogrammen erreichten „Streit um drei“ und „Barbara Salesch“ seit dem Start des RTL-Konkurrenten weniger Zuschauer. Die Seher wurden also während der betrachteten Sendungen „verloren“ und sind nicht etwa auf schwächere Vorläuferprogramme zurückzuführen. ${ }^{20}$ Die Ergebnisse weisen also darauf hin, dass der intensive Wettbewerb der Formate innerhalb kürzester Zeit zu einer Erosion dieses Wettbewerbsvorteils führt - wenn die Formate in direkter Konkurrenz stehen.

19 Hierbei wird unterstellt, dass die Formate inhaltlich sehr ähnlich sind, was angesichts der Formatstruktur (Zahl und Rollen der Protagonisten, Setting, Zahl der Fälle) und Thematiken (im Wesentlichen Strafrecht) gerechtfertigt scheint.

20 Allerdings werden nur Durchschnittswerte ganzer Sendungen betrachtet; das schlechtere Abschneiden kann deshalb auch auf qualitative Unterschiede zurückgeführt werden. Das RTLFormat „Das Familiengericht“ könnte zudem der Sendung „Barbara Salesch“ inhaltlich näher stehen als dem öffentlich-rechtlichen „Streit um drei“. Der „nur“ proportionale Verlust wäre dann auf eine starke CBBE zurückzuführen. 
Abbildung 5: Verteilung des Substitutionseffektes

\begin{tabular}{lccrc}
\hline Sendung & $\begin{array}{c}\text { Zus. } \\
\text { ab 3 }\end{array}$ & $\begin{array}{c}\text { ggü. Vor- } \\
\text { programm }\end{array}$ & $\begin{array}{c}\text { Zus. } \\
14-49\end{array}$ & $\begin{array}{c}\text { ggü. Vor- } \\
\text { programm }\end{array}$ \\
\hline „Streit um drei“ August 2002 & 0,96 & $+4,4 \%$ & 0,21 & $-4,7 \%$ \\
„Streit um drei“ September 2002 & 0,75 & $-16,9 \%$ & 0,14 & $-9,3 \%$ \\
Veränderung August/September & $-0,21$ & & $-0,07$ & \\
Erwartete Veränderung, wenn & $-0,21$ & & $-0,07$ & \\
CBBE ohne Bedeutung ist & & & & \\
„Barbara Salesch“ August 2002 & 1,98 & $+15,5 \%$ & $\% 0,86$ & $+27,7 \%$ \\
„Barbara Salesch“ September 2002 & 1,52 & $+0 \%$ & 0,60 & $-3,3 \%$ \\
Veränderung August/September & $-0,46$ & & $-0,26$ & \\
Erwartete Veränderung, wenn & $-0,46$ & & $-0,27$ & \\
CBBE ohne Bedeutung ist & & & & \\
RTL 15.00-16.00h August 2002 & 0,88 & $-20 \%$ & 0,48 & $-14,3 \%$ \\
„Das Familiengericht“ September 2002 & 1,56 & $+12,2 \%$ & 0,81 & $+17,3 \%$ \\
\hline
\end{tabular}

Quelle: Eigene Darstellung auf Basis von GfK PC\#TV.

\subsection{Vorteile des Second-to-Market}

Wie gezeigt wurde, können sich Pionierformate nur bedingt einen Vorsprung gegenüber ihren Verfolgern aufbauen. Macht dies eine entsprechende Pionierstrategie unattraktiv, können die Unternehmen, die später in den Markt eintreten, mit einigen Vorteilen rechnen. Nach Schnaars können Imitatoren zum Beispiel sicher sein, dass ein gewisses Marktpotenzial vorliegt - dies ist wegen des hohen Risikos bei der TV-Produktion von großem Wert (Schnaars 1995: 48).

Zudem kann die Strategie auch als Reaktion auf fehlende kreative Ressourcen gesehen werden. Zwar begünstigen mangelnde Programmbestände eine risikoreichere, innovativere Programmstrategie langfristig, ein Rückgriff auf die Imitation kann aber aus einer kurzfristigen Herausforderung resultieren, Sendeplätze zu besetzen. Ein Beispiel dafür war im Jahr 2002 die Umstellung der RTL-Daytime von Talk- auf Courtshows. $\mathrm{Da}$ in solchen Fällen wegen des Handlungsdrucks und drohender Konkurrenz durch andere Nachzügler wenig Zeit zur Verfügung steht, wird in der Regel versucht, das zu imitierende Produkt nach Möglichkeit nur leicht zu variieren. Schließlich kann einer Imitation auch durch einen höheren Marketingdruck zum Erfolg verholfen werden. Der ehemalige SAT.1 Leiter Fiction, Jan Kromschröder, sieht diese theoretischen Aspekte auch in der Praxis bestätigt: „Ich glaube, dass es ein Imitat auf der einen Seite leichter hat, weil das (Format, C.Z.) erlernt ist und der Zuschauer hinschaut; auf der anderen Seite auch schwerer, weil man genau hinguckt, ob das Original besser ist. Aber wenn die Imitation gewisse Qualitätsmerkmale erfüllt, dann glaube ich, dass auch ein Imitat durchaus Chancen hat."

\section{Kernkompetenzen im Programmwettbewerb}

Die Untersuchung hat deutlich gemacht, dass im Formatwettbewerb - aufgrund der häufig schnell nachlassenden Nachfrage, des hohen brancheninternen Risikos und der recht geringen Pioniervorteile - grundsätzlich eine Strategie des „Schnellen Zweiten“ zu 
bevorzugen ist, sofern es sich um Regelformate handelt ${ }^{21}$. Eine schnelle Adaptionsfähigkeit wird damit zur Kernkompetenz von TV-Sendern. Diese lässt sich in drei Dimensionen aufspalten: Absatzseitig erhöht ein starkes Senderimage - das nach Heinrich als Qualitätssignal fungiert (Heinrich 1996: 168) - die Marktchancen einer Formatinnovation. Damit gewinnt ein zum Sender passendes On-Air-Design des neuen Formats an Gewicht, um so die Senderloyalität auszunutzen. Des Weiteren kann versucht werden, die Erfolgschancen eines neuen Formats auf der Ebene der operativen Programmplanung zu erhöhen, die reliabel und längere Zeit durchzuhalten sein muss. Zubayr betont, dass eine vorhersehbare Programmplanung („Stripping“ etc.) die Einschaltquoten bei seriellen Formaten stabilisieren hilft - wenn für den deutschen Markt auch nicht von einer ausschließlich ritualisierten Fernsehnutzung auszugehen ist (vgl. Zubayr 1999).

Im Aufbau der Organisation spielt zum einen der effiziente, standardisierte Ablauf des Formatentwicklungsprozesses eine gewichtige Rolle; zum Beispiel können prozessbezogene Innovationscontrolling-Ansätze helfen, den hier entstehenden Zeitbedarf zu verringern (Schön 2001: 173). Zum anderen erlaubt eine breite Basis an Kontakten zu Produktionsfirmen/Producern bzw. das Vorhalten thematisch diversifizierter Redaktionen, flexibler auf Wettbewerbtrends zu reagieren. Insgesamt befinden sich die deutschen TV-Sender in einer turbulenten Umwelt, die zunächst das Wachstum im Gegensatz zu den 90er Jahren deutlich begrenzt und dabei wenig vorhersehbar bleibt. Zudem kann schon wegen der speziellen Struktur des Gutes „Fernsehprogramm“ und der differenzierten Rezeptionsprozesse die Komplexität der Programmentwicklung als hoch eingestuft werden. Diese Faktoren legen für den organisationalen Aufbau der (Voll-)Programme eine „Fast Generalist“-Strategie nahe, da schnell reagiert werden muss, die zukünftigen Aktionsfelder aber nur schwer prognostiziert werden können.

Schließlich kann angebotsseitig die Kooperation zwischen Sender und Produzent als zentraler Erfolgsfaktor angesehen werden. De facto verläuft die Trennlinie bei der Formatentwicklung firmenübergreifend in einem „transorganisationalen“ Nukleus (etwa von Senderredaktion und Produzent). Die Kooperation innerhalb dieser „kreativen Cluster" ist nicht durch lang laufende, formale Verträge zu erreichen, sondern durch stetig erneuerte Commitments (z. B. Aufgreifen interessanter, riskanterer Projekte; längerfristige Produktionsbeziehungen), mit der Zeit und natürlich dem Erfolg. Hier ist zu überlegen, ob Modelle der aufwandskalkulierten Vertragsgestaltung (etwa bei Auftragsproduktionen) beim Aufbau dieser Netzwerke hilfreich sind, da der Produzent in diesen Modellen seinen Gewinn an dem einzelnen Format nur dann erhöht, wenn er weniger Produktionskosten hat, als er abrechnen kann. Das Eingehen von Risiken bei einer Innovation bietet hingegen keinen finanziellen Vorteil im Erfolgsfall.

\section{Fazit}

Die TV-Sender neigen wegen der schwer prognostizierbaren Nachfrageentwicklung und des hohen Produktrisikos zu einer strukturellen Überproduktion von Formaten. Das oft beklagte „Formatfernsehen“ kann hier als Homogenisierung der Art und Wei-

21 Die Risiken der hier nicht näher betrachteten „late-to-market“-Strategie konnte man mehrfach anhand öffentlich-rechtlicher Unterhaltungsformate beobachten. Diese Sendungen wurden oftmals gestartet, als der Markt bereits gut besetzt und Sättigungstendenzen absehbar waren (z. B. Quiz- und Castingformate). 
se aufgefasst werden, wie Unterschiedlichkeit ausgedrückt wird. Mit der gestiegenen Vergleichbarkeit wird die Senderkonkurrenz vor allem zum Timing-Wettbewerb. Dahingehend ist das eingangs vorgestellte Portersche Wettbewerbskonzept um eine zeitliche Dimension erweitert worden, um auch die dynamischen Funktionen des Wettbewerbs besser erfassen zu können. Dies erscheint gerade vor dem Hintergrund der empirisch beobachtbaren Beschleunigung des Formatwettbewerbs als sinnvoll. Gerade für Inhalte außerhalb des gut untersuchten Informationsgenres fehlen bislang geeignete Instrumente, da die normative Zuschreibung von Qualität hier noch weniger möglich ist als etwa bei „harten“ Nachrichtensendungen.

Die dargestellten Erkenntnisse deuten dabei - mit Ausnahme der imagebildenden TV-Events - auf eine „Fast Second“-Strategie als optimale Unternehmensstrategie hin. Allerdings wird sich diese Forderung nicht immer in die Realität umsetzen lassen: Würden alle Unternehmen auf das zu kopierende Pionierformat warten, gäbe es keine Innovationen mehr. Zudem können TV-Unternehmen gezwungen sein, zu innovieren: Wenn eingeführte Formate langsam an Zuspruch verlieren, ohne dass eine Alternative zur Hand ist, müssen TV-Sender auch „wider Willen“ Neues wagen. Für beide Situationen - schnelle Adaption oder Innovation - lässt sich jedoch festhalten, dass Faktoren, die die Wettbewerbsflexibilität von TV-Sendern erhöhen helfen, zu zentralen Strategiegrößen avancieren.

Die Analyse unterstreicht dabei die Bedeutung der ökonomischen Konkurrenz für die Entstehung eines Qualitätswettbewerbs - zumindest außerhalb des Bereichs der „Information“. Wie gezeigt wurde, schafft das „More of the Same“ in einem Genre zugleich sein Ende und bereitet den Boden für eine neue Kategorie. In diesem Sinne erscheint eine inhaltlich-normative Forderung nach „mehr Innovation“ als unsinnig, zumal das romantische Bild der Nouveauté auf den TV-Sektor nicht anwendbar ist (Eco 1987/1994) und dementsprechend Formate als „Gesampeltes“ immer auch „Kopien“ sind. Die Programmkonkurrenz im engeren Sinne, d. h. als rein ökonomischer Wettbewerb, bietet damit nur eine schwache Legitimation für einen öffentlich-rechtlichen Rundfunk. Allerdings ignoriert dieses Wettbewerbsbild die Frage der Existenz meritorischer Inhalte (wie etwa politische Willensbildung) - was durchaus die Notwendigkeit eines Korrektivs zum Markt begründen kann. Auch wäre in weitergehenden Untersuchungen zu prüfen, inwiefern die Ergebnisse der Fallstudien auf den Programmwettbewerb - z. B. der Primetime - zu verallgemeinern wären.

\section{Literatur}

Abernathy, W. J./Utterback, P. L. (1975): A Dynamic Model of Process and Product Innovation, in: Omega, Vol. 3, 6, S. 639-656.

Arbeitsgemeinschaft der ARD-Werbegesellschaften (Hg. 2002): Media Perspektiven Basisdaten, Frankfurt a. M.

Backhaus, K. (1995): Investitionsgütermarketing, 4. Auflage, München (Vahlen).

Baumol, W. (2002): The Free-Market Innovation Machine, Princeton/Oxford (Princeton University Press).

Becker, W. /Geisler, R. (1998): Medienökonomische Grundlagen der Fernsehwirtschaft, Bamberg.

Berg, H./Rott, A. (2000): Eintritts- und Mobilitätsbarrieren im deutschen Fernsehmarkt: Das Beispiel tm3, in: Hamburger Jahrbuch für Wirtschafts- und Gesellschaftspolitik, 45, S. 317-334.

Blum, R. (1995): Television and Screen Writing, Boston u. a. (Focal Press).

Bourdieu, P. (1998): Über das Fernsehen, Frankfurt (Suhrkamp).

Brockhoff, K. (1999): Forschung und Entwicklung, 5. Auflage, München/Wien (Oldenbourg).

Brunst, K. (2003): „Nicht billig, sondern authentisch”. Interview mit Martin Hoffmann, in: epd medien, 68, S. 3-6. 
Bughin, J. (2000): Black-Scholes meets Seinfeld, in: The McKinsey Quarterly, 2, S. 13-16.

Butzek, C. (2003): Triumph der digitalen Detektivkameras, in: Medienbulletin, 7, S. 45-48.

Dehm, U./Storll, D. (2003): TV-Erlebnisfaktoren, in: Media Perspektiven, 9, S. 425-433.

Eco, U. (1987/1994): Innovation et Répetition, in: Réseaux, 68, S. 10-25.

Gitlin, T. (1994): Inside Prime Time, London (Routledge).

Groys, B. (1992): Über das Neue. Versuch einer Kulturökonomie, München (Carl Hanser).

Heinrich, J. (2002): Qualitätswettbewerb und/oder Kostenwettbewerb im Mediensektor? In: Rundfunk und Fernsehen, 2, 44, S. 165-184.

Heinrich, J. (1999): Medienökonomie, Bd. 2, Opladen (Westdeutscher Verlag).

Heinrich, J. (1996): Qualitätswettbewerb und/oder Kostenwettbewerb im Mediensektor, in: Rundfunk und Fernsehen, 44, S. 165-184.

Hesse, M. (2002): Frauen-Fernsehen und Fernseh-Frauen, in: Fernseh-Informationen, 4/2002.

Hickethier, K. (2000): Fernsehen. Modernisierung und kultureller Wandel, in: Flach, S. /Grisko, M. (Hg.): Fernsehperspektiven, München (KoPäd-Verlag), S. 18-36.

Höltich, L.-U. (1994): Die Daytime bei RTL plus - eine Betrachtung unter programmlichen und finanziellen Gesichtspunkten, in: Bosshart, L./Hoffmann-Riem, W. (Hg.): Medienlust und Mediennutz, München (Ölschläger), S. 367-374.

HMR International (2002): The German TV-Market Inside-out, Köln.

Hofmann, B. /Rauscher, M. (1991): Complex Dynamics in Fashion Life Cycles, Kiel (Kieler Arbeitspapiere, Nr. 486).

Holtmann, K. (1999): Programmplanung im werbefinanzierten Fernsehen: Eine Analyse unter besonderer Berücksichtigung des US-amerikanischen Free-TV, Lohmar/Köln (Eul).

Holtmann, K. (1998): Programmbeschaffung und -entwicklung werbefinanzierter TV-Programmanbieter aus der Perspektive der Programmplanung, Köln (Arbeitspapiere des Instituts für Rundfunkökonomie Nr. 106).

Holzer, D. (1999): Die deutsche Sitcom, Bergisch-Gladbach (Lübbe).

Holzporz, S. (2002): Der rechtliche Schutz des Fernsehshowkonzeptes, Münster (Lit).

Kauschke, A. /Klugius U. (2000): Zwischen Meterware und Maßarbeit, Gerlingen (Bleicher).

Keller, K. L. (1998): Strategic Brand Management, New Jersey (Prentice-Hall).

Keller, K. L. (1993): Conceptualizing, Measuring, and Managing Customer-Based Brand Equity, in: Journal of Marketing, 57, S. 1-22.

Kennedy, R. E. (2002): Strategy fads and competitive convergence, in: Journal of Industrial Economics, $1,57-85$.

Kotzbauer, N. (1992): Erfolgsfaktoren neuer Produkte, Frankfurt/M. u. a. (Lang).

Krüger, U. M. (2001): Die Boulevardisierungskluft im deutschen Fernsehen, in: Media Perspektiven, 7, S. 326-344.

Krüger, U. M. (2000): Programmprofile im deutschen Fernsehen 1991-2000, Baden-Baden (Nomos).

Kruse, J. (2000): Ökonomische Probleme der deutschen Fernsehlandschaft, in: Ders. (Hg.): Ökonomische Perspektiven des Fernsehens in Deutschland, Hamburg, S. 7-47.

Litman, B./Shrikhande, S./Ahn, H. (2000): A Portfolio Theory Approach to Network Program Selection, in: Journal of Media Economics, 13, 2, S. 57-79.

Ludwig, J. (1998): Zur Ökonomie der Medien: Zwischen Marktversagen und Querfinanzierung, Opladen (Westdt. Verlag).

Mann, C. (2004): Formatting for Success, in: Advanced Television, www.advanced-television.com (10. 5. 2004).

McDowell, W./Sutherland, J. (2000): Choice versus Chance: Using Brand Equity Theory to Explore TV Audience Lead-In Effects, A Case Study, in: Journal of Media Economics, 4, 13, S. 233-247.

Meckel, M. (1997): Die neue Übersichtlichkeit, in: Rundfunk und Fernsehen, 4, 45, S. 475-485.

Miller, D. /Shamsie, J. (1999): Strategic Responses to Three Kinds of Uncertainty: Product Line Simplicity at the Hollywood Film Studios, in: Journal of Management, 1, 25, S. 97-116.

Neumann, R. (1991): The future of the mass audience, Cambridge (Cambridge university press). 
o. V. (2001): „No Risks, No Profits“ Interview mit SAT. 1 Chef Martin Hoffmann, in: Medien Bulletin, 6, S. 38-45.

o. V. (2001a): TV/Formate/Marktanteile, in: Mediafacts, 2, S. 16-20.

o. V. (2002): Mit Ruhe und Gelassenheit ans Ziel - Axel Beyer über Zeitgeist, Comedy \& neue TVFormate, in: Medien Bulletin, April 2002, S. 48-57.

Pätzold, U. /Röper, H. (2002): Film- und Fernsehproduktionsmarkt Deutschland. o. O.

Pethig, R. /Blind, S. (1995): Programmformatentwicklung im Wettbewerbsprozess: Innovationsund Imitationszyklen, in: Hallenberger, G. (Hg.): Neue Sendungsformen im Fernsehen, Siegen, S. 57-77.

Pleschak F./Sabisch H. (1996): Innovationsmanagement, Stuttgart (Schaeffer-Poeschel).

Porter, M. E. (1999): Wettbewerbsstrategie, 10. Aufl., Frankfurt/New York (Campus).

Röper, H. (2000): Zur Lage mittelständischer Fernsehproduzenten in Deutschland, Dortmund.

Rott, A. /Schmitt, S. (2001): Wirkungen von Programmereignissen auf die Zuschauernachfrage. Eine empirische Analyse am Beispiel von „Wetten, dass ...? “ in: Media Perspektiven, 5, S. $258-263$.

Rott, A. (2003): Werbefinanzierung und Wettbewerb auf dem deutschen Fernsehmarkt. Berlin (Duncker \& Humblot).

Rust, H. (1988): Imitation als Programmkonzept, in: Media Perspektiven, 10, S. 611-620.

Schmidt, S. J. (2000): Werte-Rohstoff, in: epd medien, 84, S. 5-10.

Schnaars, S. P. (1995): Pioniere überflügeln, Freiburg (Rudolf Haufe).

Schön, A. (2001): Innovationscontrolling, Frankfurt/Berlin u. a. (Peter Lang).

SevenOneMedia (2002): TV-Images 2002, Unterföhring.

Stigler, G./Becker, G. (1977): De Gustibus Non Est Disputandum, in: American Economic Review, 67, S. 76-90.

Winter, M. (1999): Programmbeschaffungsmarketing privater Fernsehveranstalter, Marburg (Tectum).

Wolf, F. (2003): Alles Doku - oder was? Über die Ausdifferenzierung des Dokumentarischen im Fernsehen. Düsseldorf (LfM-Dokumentation Band 25).

ZDF (2002): Fernsehsender im Urteil der Zuschauer, Mainz.

Zubayr, C. (1999): The Loyal Viewer? Patterns of Repeat Viewing in Germany, in: Journal of Broadcasting \& Electronic Media, 3, 43, S. 346-363.

\section{Anhang: Liste der Interviewpartner}

- Matthias Alberti, Bereichsleiter Unterhaltung SAT.1

- Jobst Benthues, Leitung Unterhaltung ProSieben Television GmbH

- Martin Berthoud, Leitung Programmplanung, ZDF

- Borris Brandt, General Manager Endemol

- Klaudia Brunst, Fernsehkritikerin, ehem. Mitglied der Jury des Deutschen Fernsehpreises

- Marc Conrad, Geschäftsführer Typhoon Networks AG

- Sabine Eckhard, Head of Serial Production, UFA-Fernsehproduktion GmbH

- Harry Goering, Geschäftsführer UFA Entertainment GmbH

- Jörg Grabosch, CCO Brainpool TV AG, Vorstand Content der Viva Media AG

- Gebhard Henke, Leiter Programmbereich Fernsehfilm, WDR

- Nico Hofmann, Geschäftsführer teamworx Produktion für Kino und Fernsehen GmbH

- Jan Kromschröder, ehem. Leiter Fiction, SAT.1

- Friedrich Küppersbusch, Geschäftsführer ProBono TV

- Christiane Ruff, Geschäftsführerin Sony Pictures Entertainment Deutschland

- Tom Sänger, Leiter Unterhaltung RTL

- Jan-Richard Schuster, Abteilungsleiter Serien, RTL

- Norbert Schneider, Direktor der Landesanstalt für Medien NRW 